\title{
English for specific purposes (ESP) et français sur objectifs spécifiques (FOS) : le préalable du contenu préalable
}

\section{Denis Lehmann}

\section{(2) OpenEdition \\ Journals}

Édition électronique

URL : http://journals.openedition.org/asp/2938

DOI : 10.4000/asp.2938

ISSN : 2108-6354

Éditeur

Groupe d'étude et de recherche en anglais de spécialité

\section{Édition imprimée}

Date de publication : 1 décembre 1997

Pagination : 11-26

ISSN : 1246-8185

\section{Référence électronique}

Denis Lehmann, «English for specific purposes (ESP) et français sur objectifs spécifiques (FOS) : le préalable du contenu préalable », ASp [En ligne], 15-18 | 1997, mis en ligne le 16 avril 2012, consulté le 19 avril 2019. URL : http://journals.openedition.org/asp/2938; DOI : 10.4000/asp.2938

Ce document a été généré automatiquement le 19 avril 2019

Tous droits réservés 


\title{
English for specific purposes (ESP) et français sur objectifs spécifiques (FOS) : le préalable du contenu préalable
}

\author{
Denis Lehmann
}

1 Redoutable honneur, pour un non-angliciste, que de tenter ici la synthèse entre ce qui rapproche et ce qui sépare les conceptions britanniques et françaises en matière d'enseignement des langues étrangères aux publics dits "spécifiques». Mais si j'en ai accepté le risque c'est que, dans ce secteur de la didactique des langues, un spécialiste de français langue étrangère ne peut aujourd'hui faire l'économie de suivre à la jumelle ce qui se passe et s'écrit à l'autre bout du tunnel ${ }^{1}$.

2 Je me dis aussi que la mise en balance de ces deux traditions revient à comparer l'éléphant et la puce ; et que ma préférence avouée pour le plus gros des deux représente à coup sûr un atout décisif. Car la première différence entre l'English for Specific Purposes (dorénavant ESP) et le Français sur Objectifs Spécifiques (ci-après FOS) est évidemment de nature quantitative: ici un marché de formation linguistique assez restreint, d'un dynamisme grandissant mais encore limité, auquel ne correspond qu'un marché éditorial étroit, ne comptant au surplus qu'une maigre poignée d'auteurs potentiels; là un marché de formation immense, ouvrant des perspectives non négligeables à de puissants éditeurs alimentés par nombre d'équipes, institutions et auteurs ayant pignon sur rue.

3 Si le bilan de ce qui nous unit et de ce qui nous sépare va principalement nous conduire à ausculter la sphère des théorisations didactiques et des choix méthodologiques subséquents, il me semble utile de porter sur la question deux brefs éclairages préalables qui touchent d'abord au poids tout à fait particulier qu'exercent les réalités extradidactiques (politiques, économiques et institutionnelles) sur l'activité didactique en FOS, 
puis à la cartographie comparée des domaines, disciplinaires et professionnels, de cette activité dans les deux champs.

\section{L'influence des réalités politico-économico- institutionnelles en FOS}

Depuis la Seconde Guerre mondiale au moins, la diffusion de sa langue et de sa culture a représenté pour la France une «affaire d'État », si l'on veut bien considérer l'expression dans ses deux sens.

5 1.1. À la différence de la plupart des autres pays nourrissant les mêmes ambitions, des institutions d'État sont en charge de cette politique et d'une partie significative de sa mise en oeuvre : singulièrement les ministères des Affaires étrangères et de la Coopération, par le truchement des Services culturels de nos ambassades et des organismes qui en dépendent, soit en propre (Services de Coopération Linguistique et éducative des Instituts et Centres culturels), soit indirectement (les Alliances françaises et leur réseau ; l'Agence pour l'Enseignement Français à l'Étranger, qui régit les écoles et lycées français); sans oublier ni la part que prend le ministère de l'Éducation dans ce concert ni, plus sujettes à variation et plus pianissimo, celles des ministères en charge de la culture ou de la francophonie.

6 1.2. Plus significatif encore sans doute, le fait que, de tous les pays dits industrialisés, ce soit le nôtre qui consacre le plus fort pourcentage du budget de l'État à la diffusion de sa langue et de sa culture. Par delà la baisse tendancielle et probablement irréversible de ce pourcentage, on a pu naguère observer une étroite corrélation entre, d'un côté, la chronologie des « chocs pétroliers » et, de l'autre, les « dents de scie » qui affectent cette baisse et qu'accompagnent, avec une régularité quasi métronomique, les virages (parfois à cent quatre-vingts degrés) des politiques de diffusion culturelle et linguistique.

7 Mieux encore peut-être qu'une typologie de paramètres constituant l'éventail des possibles en matière de politiques linguistiques, de politiques éducatives et de politiques de diffusion, les quelques propos que voici ${ }^{2}$ - propos de plume comme de fins de repas, d'ailleurs - illustrent, jusque dans le prosaïsme des uns et le cynisme de quelques autres, la prégnance qu'exercent sur le FOS les sphères économique, politique et institutionnelle ${ }^{3}$ (voir figure 1). 
1. «La francophonie ouvre à la France un marché potentiel de 150 millions de consommateurs.»

2. "S'agissant de l'image que nous donnons, pouvons-nous échapper à la fatalité qui nous condamne à naviguer entre la culture classique (type Lagarde et Michard), le cocorico technologique (type T.G.V.) et les délices de Paris (type Coco Chanel) ?»

3. «Au-delà de la scolarisation, c'est l'absence d'un environnement culturel francophone qui constitue le plus souvent l'obstacle principal à une diffusion efficace de notre langue. »

4. «Marcel Dassault ou Victor Hugo?»

5. «Ce dont nous avons besoin c'est de la langue française et non de sa culture.»

6. «Peu importe la langue, ce que nous exportons c'est la France. »

7. « Mettre l'accent sur nos points forts et non sur nos faiblesses. »

8. "Là où le français est distancé de façon irréversible, on limitera les objectifs à des auditoires spécialisés en liaison avec nos opérations de coopération économique, technique et scientifique.»

Bien entendu, les choix politiques ainsi affichés manquent rarement, pour peu qu'ils émanent de décideurs, de se traduire en termes sonnants et trébuchants venant rythmer les fluctuations en moyens investis par la sphère politique française au service de ces choix. Le constater n'est pas feindre d'ignorer que l'enseignement de toute langue étrangère est avant tout l'affaire de chaque pays, de ses propres décideurs, enseignants, apprenants. Ainsi du «frances instrumental » en Amérique Latine : après avoir suscité les travaux sur la méthodologie d'apprentissage de la lecture entrepris par plusieurs équipes françaises à la fin des années soixante-dix, il n'a guère été affecté par les avatars de la politique de diffusion française.

\section{L'éventail des secteurs d'intervention}

Consultation des catalogues de grands éditeurs britanniques ou fossé quantitatif évoqué tout à l'heure, tout donne à penser que l'ESP couvre probablement au mieux l'éventail des disciplines, domaines, métiers et professions, sans que pour autant cette couverture ait partout la même épaisseur. Il n'en va pas du tout de même s'agissant du FOS.

\subsection{Disciplines, professions, métiers}

À se pencher sur les deux indicateurs que constituent la production éditoriale de manuels et les diplômes de langue spécialisée, on aboutit sensiblement au même diagnostic : seuls deux domaines du FOS tirent à peu près leur épingle du jeu. Le "français des affaires", bien doté en manuels, souvent récents, nantis en proportion croissante de supports électroniques réputés interactifs (bande vidéo, CD-Rom); il doit une bonne part de son dynamisme à l'action de la Chambre de Commerce et d'Industrie de Paris et à son réseau planétaire, tissé par association avec une multitude de centres d'enseignement qui trouvent leur intérêt dans la préparation aux certificats de français spécialisé de la CCIP. 
Le «français du tourisme " bénéficie lui aussi, bien qu’à moindre titre, des mêmes conditions de diffusion.

11 Quant aux travaux universitaires ayant donné lieu à production ou valorisation pédagogique, ils concernent très majoritairement le secteur des Sciences humaines et sociales.

12 À cette maigre moisson, on peut ajouter quelques propositions de topographie ${ }^{4}$ et deux enquêtes déjà anciennes, livrées à l'initiative du Ministère des Affaires étrangères entre 1987 et 1990. Des analyses menées par J. Couillerot et H. Farid (1990) sur la seconde livrent les enseignements significatifs (voir figure 2).

Figure 2

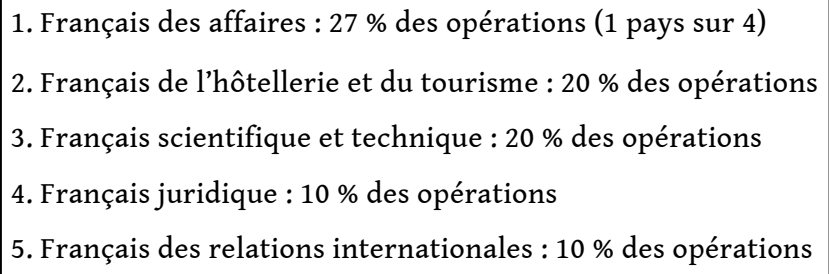

\subsection{Publics : professionnels ou étudiants?}

Si la distinction - traditionnelle en ESP - entre «Occupational Purposes » et "Academic Purposes » a pu s'incarner naguère en FOS de façon assez exemplaire dans de vastes opérations de mise à niveau linguistique connues sous l'appellation de «boursiers prioritaires $»^{5}$ force est de reconnaître que le marché éditorial persiste à ignorer superbement ${ }^{6}$ le «Français sur Objectifs Académiques" et que nombre d'étudiants étrangers continuent à connaître l'échec dans leurs études en France pour des raisons essentiellement linguistiques. À l'aune des résultats bruts de l'enquête MAE, il pourrait sembler qu'il en aille autrement hors de France, où près de $50 \%$ des opérations recensées concernent des étudiants (voir figure 3).

Figure 3

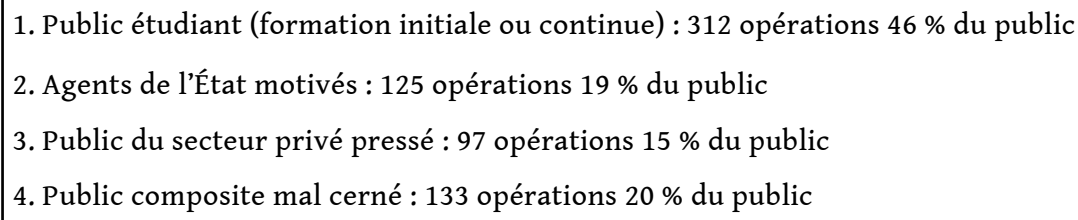

Du moins serait-on porté à le croire, si un discret constat de la même étude ne révélait qu'une grosse moitié des opérations recensées sous l'étiquette FOS désignent en fait des cours de langue usuelle, qui n'ont de spécifiques que de rassembler ici des étudiants médecins et là des étudiants juristes. Déplorable réalité qui caractérise aussi, semble-t-il, bon nombre des nouveaux cours de «langue pour non-spécialistes» de nos propres universités. 


\subsection{Capacité/habileté/compétence}

Dernière particularité notable du FOS, surtout en contexte universitaire étranger : un assez important déséquilibre en faveur de l'écrit et particulièrement de la compréhension écrite, du fait de choix «stratégiques " privilégiant, aux fins de développement, l'accès à la bibliographie scientifique, technologique et professionnelle en français. C'est ce que l'on nomme «frances instrumental » en Amérique latine, option aujourd'hui largement répandue et dont on ne peut que se féliciter :

16 (i) elle constitue une réponse fonctionnelle à des besoins dotés de quelque semblant de réalité ; autrement dit, si des sources bibliographiques en français sont à la portée des étudiants concernés, ce qui est loin d'être toujours le cas; ou qu'elle ne se trouve pas dictée par la dérisoire étroitesse des masses horaires consenties aux langues étrangères dans les cursus universitaires bien plus que par des besoins réels ;

(ii) à la condition, enfin, qu'elle n'ouvre toute grande la porte aux plus antédiluviennes des pratiques: version et explication de texte notamment, parées à l'occasion des vertueux atours de l'éclectisme.

\section{Présupposés théoriques et conceptions didactiques}

Nous voici donc, avec ces dernières considérations, à la charnière entre caractéristiques des publics et nature de la construction méthodologique proposée en réponse. C'est maintenant aux aspects proprement didactiques que sera consacrée la poursuite de ce qui rapproche ou, à l'inverse, sépare le FOS et L'ESP. Deux remarques préliminaires s'imposent néanmoins.

19 Il est patent qu'en matière d'innovation méthodologique le vent souffle de l'ouest depuis pas mal d'années. Si les revues britanniques citaient volontiers les spécialistes français au plus fort de la vague structuro-globale-audio-visuelle et des «langues de spécialité », il n'en va plus du tout de même aujourd'hui, où les flux se sont totalement inversés; certains voudraient n'y voir qu'un effet secondaire des travaux menés sous l'égide du Conseil de l'Europe, exonérant à trop bon compte les didacticiens du Français langue étrangère de leur dynamisme perdu.

D'autre part, ce que je vais présenter maintenant pourrait donner à croire qu'ESP et FOS seraient en quelque sorte monolithiques; simple illusion qui ne tiendrait alors qu'au mode de présentation adopté (Tableau 1), où l'expéditif le dispute au lapidaire un peu plus que de raison ; y sont schématisés, en lignes et en colonnes :

21 (i) trois tendances méthodologiques «canoniques": loin d'être majoritaires ou dominantes dès leur apparition, échelonnée sur une longue période (ce qui ne les empêche nullement de cohabiter aujourd'hui encore), elles ont seulement représenté alors le courant «moderniste», alternatif, novateur, celui par rapport auquel les autres courants ont été amenés à se situer ;

(ii) les fortunes diverses rencontrées selon moi, respectivement au sein de l'ESP et du FOS, par chacune de ces trois tendances ;

23 (iii) enfin, le point de vue qui est le mien aujourd'hui sur chacune d'elles, et qui ne peut évidemment se confondre avec l'état du FOS, ni moins encore lui tenir lieu de doctrine. 


\subsection{Panorama schématique : trois tendances majeures et canoniques}

Tableau 1

\begin{tabular}{|c|c|c|c|}
\hline & $\begin{array}{l}\text { ESP } \\
\text { (panorama schématique et } \\
\text { subjectif) }\end{array}$ & $\begin{array}{l}\text { FOS } \\
\text { (panorama schématique et } \\
\text { subjectif) }\end{array}$ & $\begin{array}{l}\text { FOS } \\
\text { (point de vue personnel) }\end{array}$ \\
\hline 1 & $\begin{array}{l}\text { Linkstiks and Philogs } \\
=\text { de AL à Applied DA }\end{array}$ & $\begin{array}{l}\text { des survivances notables } \\
=\text { résidus grammaire } \\
\text { traduc./ langues de } \\
\text { spécialité= AD appliquée }\end{array}$ & $\begin{array}{l}\text { décidément NON, mais ... } \\
\text { places et fonctions de } \\
\text { l'intervention linguistique }\end{array}$ \\
\hline 2 & $\begin{array}{l}\text { Pr Trim and Dr Soandso } \\
=« \text { système » européen } \\
\text { «SABOC» }\end{array}$ & $\begin{array}{l}\text { plutôt « rétro" mais } \\
\text { "tendance» }\end{array}$ & $\begin{array}{l}\text { OUI oui }=>\text { oui } \text { MAIS => } \\
\text { finalement NON : critique du } \\
\text { modèle «SABOC " }\end{array}$ \\
\hline 3 & $\begin{array}{l}\text { Sing along with Lancaster! } \\
=\text { priorité aux modalités de } \\
\text { l'apprentissage = contenu : } \\
\text { non linguistique/pas de } \\
\text { contenu préalable }\end{array}$ & $\begin{array}{l}\text { plutôt NON } \\
\text { (« restons français! » donc } \\
\text { cartésiens ...) }\end{array}$ & $\begin{array}{l}\text { OUI et NON, ENCORE que : } \\
\text { besoin des besoins, } \\
\text { diversifier les } \\
\begin{array}{ll}\text { contenus,importance des } \\
\text { modalités de l'apprentissage }\end{array}\end{array}$ \\
\hline
\end{tabular}

\section{Commentaires}

Linkstiks § Philogs : On pardonnera des facéties qui ne seraient que de potache si elles ne se voulaient un hommage moins discret que sincère à certains travaux (publiés ou non, je ne l'ai jamais su) de Tom Hutchinson concernant les représentations que les enseignants de langue se font de leur métier. Heureuse coïncidence, Hutchinson est bien connu des anglicistes pour des positions novatrices, en matière d'ESP notamment (Hutchinson $\S$ Waters, 1987), qui sont par ailleurs la marque de son université7. Les qualificatifs utilisés par notre auteur (j'ose à peine, ici, souligner l'allusion transparente aux sinistres Bandarlogs) ne laissent planer aucun doute sur son opinion concernant les tendances de type "linguistique appliquée». Bien que stigmatisées de longue date par nombre de linguistes et didacticiens de toutes langues, elles n'en continuent pas moins à prospérer, $\mathrm{y}$ compris dans les didactiques de nos deux langues ${ }^{8}$, qu'elles empruntent ou non la forme particulière et teintée de modernisme d'une analyse de discours appliquée. De l'autre bord d'un affrontement stérile et très franco-français, le rejet de la linguistique appliquée dégénère trop souvent en rejet de la linguistique dans son entier, là où l'on rêve de n'y trouver que refus de toute logique de disqualification, de refoulement, de l'affrontement boutiquier, en somme de tout ce qui, non dit, se pourrait exprimer en ces termes: «les seules questions que je peux traiter sont des questions d'ordre linguistique; donc, seules sont dignes d'intérêt les questions relevant de la linguistique; toute autre est frappée de nullité, problème qui n'existe pas!».

Professor Trim and Doctor Soandso : Ici, chacun aura reconnu ce courant novateur qui, sous l'impulsion du Conseil de l'Europe à la charnière des années 60 et 70 va marquer 
durablement la didactique des langues étrangères, en Europe et bien au-delà. La référence à John Trim, infatigable animateur des groupes d'experts, ne surprendra personne ; elle veut aussi souligner le statut de «balise» tenu par le document de 1973 (Trim, ed.), premier jalon largement diffusé en préliminaire à l'élaboration successive des niveauxseuils. Rappelons que s'y trouvent rassemblés les principes essentiels du système européen en gestation : à côté des modes d'organisation généraux (place des niveauxseuils dans un syllabus constitué d'unités capitalisables notamment), trois contributions articulées donnent à voir la philosophie du projet en ce qu'elle représente de plus innovant :

(i) Celle de R. Richtérich marque l'irruption, en alternative à l'ancienne « centration sur le contenu », d'une première forme de " centration sur l'apprenant », par la prise en compte de l'extrême diversité

- des situations d'apprentissage (S),

- des publics d'apprenants visés (A),

- de leurs besoins langagiers (B) liés à des situations cibles également très diverses,

- le tout devant déboucher sur la formulation d'objectifs d'apprentissage (0).

Une telle approche concrétise en même temps l'émergence d'un regard de nature sociologique dans la didactique des langues, qu'il contribue ainsi largement à fonder.

(ii) Celle de D. Wilkins complète le modèle de traitement des demandes de formation linguistique dans sa dernière étape, l'élaboration du contenu langagier d'apprentissage (C) ${ }^{9}$. Ce modèle s'incarne ensuite dans un texte de Van Ek, présentant le Threshold Level dont il est le concepteur et qui viendra bientôt inaugurer la série des niveaux-seuils. Ici, deuxième « révolution » didactique, de nature linguistique cette fois : rendue possible par les évolutions de cette discipline dans les années soixante et appelée aussi bien par un Widdowson que par un Roulet, elle invite à se tourner vers ce que ce dernier nommait les «linguistiques de l'usage». S'agissant des unités linguistiques de contenu, elles s'incarnent chez Wilkins en " notions » et « fonctions », cadre dont s'inspire, en partie du moins, le Threshold Level. Marquant doublement leur différence, les concepteurs de la version consacrée au français ${ }^{10}$, qui paraît l'année suivante, placeront Austin, Searle et la théorie des actes de parole (ou de langage) au coeur d'un appareil dont le titre, Un niveauseuil, donne à voir, non sans un soupçon d'ostentation, qu'il se veut une simple proposition jetée à l'océan des possibles.

Le sort qui sera réservé ici et là au projet européen dans la seconde moitié des années soixante-dix diffère évidemment sur bien d'autres points. S'agissant du système dans son ensemble, à une adhésion somme toute modérée ne répond, du côté du FOS, qu'une critique elle-même timide : si, quant au principe, la prise en compte des besoins fait tant bien que mal son chemin, les outils d'identification restent le plus souvent au placard. Par contre, c'est sur le terrain linguistique (conception de Un niveau-seuil et recours à la théorie des actes de parole) que se concentre une contestation (parfois violemment polémique) issue de divers courants, notamment: chercheurs du CRAPEL de Nancy, proches (et d'ailleurs traducteurs) de T.C. Jupp ; têtes pensantes et agissantes de la revue $D R L A V$, à l'Université Paris 8 ; linguistes et didacticiens de l'Université Paris 7 se réclamant de la théorie d'Antoine Culioli ${ }^{11}$. Pour ce qui est de la didactique de l'anglais langue étrangère et de l'ESP, à une adhésion d'autant plus massive qu'elle est plus aisée (la formation continue y est d'implantation plus ancienne et les nouvelles linguistiques de référence beaucoup mieux connues, puisque majoritairement 
autochtones) font écho des réactions de rejet qui n'en seront que plus vives (à la fois plus rapides et plus tonitruantes), plus globales, et infiniment plus radicales.

Sing along with Lancaster: Facilité de présentation encore, dans la troisième strate du tableau 1, que d'avoir réservé la vedette aux gens de Lancaster, marquant ainsi l'impact persistant de ce qui s'est fait là ; nul n'oublie pour autant que, parmi les didacticiens de l'anglais, il s'en trouva bien d'autres à tailler des croupières au Conseil de l'Europe et à ses épigones ${ }^{12}$. Quant à ce sing along, il claironne ${ }^{13}$ le retour en grâce des modalités de l'apprentissage, noeud gordien d'un nouveau virage méthodologique qui fait suite à une décennie très profondément marquée par le modèle du Conseil de l'Europe.

\subsection{Le préalable du contenu préalable}

\subsubsection{Trois modes de gestion de la diversité}

31 L'alternative à trois termes qui vient dessiner mon horizon didactique trouve sa rationalité dans la spécificité essentielle - peut-être la seule - d'un domaine qui, par ailleurs, colle à une histoire récente de la didactique des langues dont elle épouse assez exactement les courants majeurs; spécificité qu'en sociologie des organisations l'on nommerait un problème de "gestion de la complexité ", et qui est surtout un problème de gestion de la diversité. La parenté entre FOS/ESP et Formation continue est ici assez évidente. Ces deux champs ont en effet en commun d'avoir à gérer une infinité de critères diversificateurs, là où les systèmes éducatifs scolaires ne se donnent le plus souvent à traiter, en fait de paramètres, que l'âge des apprenants-élèves, traduit dans la succession des cycles et des classes, au long d'un cursus fort linéaire. Ce dispositif est seulement complexifié en bout de course par la répartition des élèves au sein de quelques segments parallèles, faisant alors intervenir plus explicitement des paramètres de contenu.

Face au défi de la diversité/complexité, l'attitude des didacticiens des langues a consisté à choisir un angle privilégié de traitement, modalité que l'on avait pris l'habitude, en Français langue étrangère du moins, de désigner du terme de " centration ». Le Tableau 2 ci-dessous schématise les trois types de centration qui peuvent être envisagés et qui représentent autant de tendances canoniques, correspondant à la fois :

- aux trois questions classiques : « quoi ? ", « à qui ? » et « comment? »

- aux trois objets constitutifs de l'activité d'enseignement/apprentissage, ou plus précisément à une construction didactique opérée sur ces trois objets

- aux trois strates « de fantaisie » du tableau 1.

Tableau 2

\begin{tabular}{|l|l|l|l|}
\hline & type de centration & paramètre privilégié \\
\hline $\begin{array}{l}\text { 1. Linguistique } \\
\text { appliquée }\end{array}$ & $=$ & «sur le contenu » & la langue/le discours \\
\hline $\begin{array}{l}\text { 2. Système européen } \\
\text { S - A - B - O - C }\end{array}$ & $=$ & "sur l'apprenant » & $\begin{array}{l}\text { les besoins langagiers } \\
(=\text { les situations cibles })\end{array}$ \\
\hline
\end{tabular}




\begin{tabular}{|l|l|l|}
\hline $\begin{array}{l}\text { 3.1 Contenu non } \\
\text { linguistique } \\
\text { ou } \\
\begin{array}{l}\text { 3.2. Pas de contenu } \\
\text { préalable }\end{array}\end{array}=\begin{array}{l}\text { les opérations cognitives } \\
\text { les pratiques de classe : tâches ou activités } \\
\text { communicatives, } \\
\text { l'apprentissage » }\end{array}$ \\
\hline
\end{tabular}

\subsubsection{Introuvable apprenant}

33 La deuxième tendance, éclose avec les années soixante-dix sous les couleurs d'un changement de " centration » avait, en réalité, pour caractéristique et, surtout, pour effet imprévu de renouveler les conditions de détermination des contenus d'enseignement eux-mêmes, encore et toujours posés comme préalable à toute réponse adéquate au défi de la diversité des publics demandeurs de formation linguistique. En somme, outre divers autres défauts, elle m'apparaît aujourd'hui comme une fausse «centration sur l'apprenant ", une "centration sur le contenu » maintenue et qui ne dit pas son nom; point de vue, on le voit, qui n'est guère différent de celui exprimé naguère par Hutchinson et Waters (1987). En résumé :

34 - Réduction, refoulement du sujet : l'apprenant n'y est pris en compte que d'un unique point de vue, auquel se trouve presque toujours réduite l'analyse : celui de ses besoins supposés; à ce titre, il est bien difficile de considérer que l'apprenant soit véritablement appréhendé en tant que «sujet», dans ses dimensions aussi bien sociales que psychologiques.

35 - Idéalisation : à y regarder de plus près, ces besoins que l'on s'attache à identifier minutieusement ne sont pas les siens; j'entends par-là que si Carla Lambda, hôtesse de l'air, et Peter Soandso, steward, souhaitent suivre un cours de FOS en vue d'affronter le légendaire monolinguisme des voyageurs français, ce ne sont pratiquement jamais leurs propres besoins qui servent de référence mais ceux, postulés, d'un «sujet-hôtesse/ steward-locuteur-idéal » parlant un «français-idéal » dans des «situations-decommunication-idéales » avec les «sujets-passagers-locuteurs-idéaux » des "avionsidéaux » d'une « compagnie-idéale ».

36 - Paris (risqués) et stéréotypes (tenaces) : ce qui vient d'être dit n'est que l'effet pervers d'une problématique opératoire qui semblait a priori prometteuse, celle des situations cibles. Mais le rôle de baromètre des contenus qui lui est attribué n'autorise qu'un seul jeu: celui des "comme si» (comme si tout le monde avait les mêmes habitudes et comportements culturels, réagissait de la même manière, parlait de la même manière, apprenait de la même manière, etc.), aux antipodes d'une centration vraie sur les spécificités des apprenants. Les risques du pari se cumulent avec celui, maintes fois observé, de ne voir retenues que les plus prévisibles et les plus stéréotypées de ces situations, celles où tout énoncé produit est correct, où tout énoncé produit est compris ; et où il $\mathrm{y}$ a toujours un pilote dans l'avion...

37 - Déplacement de perspective : au bout du compte, parti d'une problématique de nature sociologique destinée à interroger la diversité des publics d'apprenants, on en revient subrepticement à une problématique linguistique (et, dans le meilleur des cas, sociolinguistique) où les deux seules questions qui vaillent sont: (i) quelles sont les formes linguistiques observables dans un corpus de situations de communication 
désignées comme cibles? (ii) Lesquelles de ces formes doivent être acquises par un alloglotte désireux de communiquer efficacement dans de telles situations?

Ceci étant, le rejet d'une telle démarche ne justifie en aucune manière que l'on puisse conclure - et certains ne s'en sont pas privés - à l'inutilité, voire à la nocivité, d'une quelconque prise en compte des besoins. Il ne disqualifie ni les outils d'identification, ni moins encore l'utilité et l'usage de la notion de situation cible. Ce qui est ici en question, c'est l'hyper-linéarité mécanique du système SABOC conduisant à l'hypertrophie des effets pervers, l'enchaînement univoque de relations de cause à effet sous-tendant une séquence immuable d'opérations didactiques posées comme canoniques et, au total, la traduction-réduction, immédiate et imprudente, de chacun des constituants à celui qui le suit (voir figure 4).

\section{Figure 4}

Situation $=$ Apprenant $=$ Besoins $=$ Objectifs $=$ Contenus (langagiers d'apprentissage)

On devrait également se garder d'en conclure sans plus d'examen qu'une véritable centration sur l'apprenant demeure hors de nos prises. Remarquons simplement ceci : quelle que soit la langue étrangère en question, c'est du côté de l'apprentissage en autonomie que semble se poursuivre aujourd'hui en France sa quête la plus active. Et craignons, par-dessus tout, qu'en dépit de ses évidents avantages, cette formule ne soit bientôt plus que le dernier des miroirs aux alouettes, si l'on ne s'y donne les moyens de gérer au plus près la diversité des représentations, habitudes et comportements d'apprentissage; on ne pourrait alors que succomber à ce même fléau qui mine la centration sur l'apprenant dans une pratique de cours ordinaire.

\subsubsection{La France est-elle une île?}

41 Enfin, l'alternative qui voit le jour au tournant des années quatre-vingt entend renverser l'ordre des priorités didacticiennes en faveur des modalités de l'apprentissage, grandes absentes du projet européen. Souvent présenté comme un abandon du «quoi enseigner/ apprendre? » au profit d'un " comment enseigner/apprendre? », ce virage est cependant assez loin de se négocier dans un ordre aussi parfait. Ce qui, aux yeux de quelques-uns, marque le passage à une deuxième génération des approches communicatives venant tout naturellement redresser les balbutiements de la première sera salué, ailleurs, comme l'avènement d'une ère didactique nouvelle, parfois affublée du nom de "postcommunicatif » :

(i) D'un côté, certains didacticiens - anglo-saxons surtout, de Lancaster ou d'ailleurs - se font les champions de l'inutilité, voire de la nocivité du « quoi enseigner/apprendre ? » et en concluent qu'il n'y a pas lieu de se préoccuper plus avant d'élaborer des contenus préalables (le 3.2. du Tableau 2) ; pour eux, le contenu ne sera tout au plus que le relevé $a$ posteriori de ce qui s'est passé dans un cours.

43 (ii) Mais il en est d'autres, également didacticiens de l'anglais langue étrangère, pour ne mettre en question que le contenu même du contenu préalable, et non pas son principe : ils proposent alors des contenus et des syllabus (contenus organisés en progression) qui ne sont plus constitués d'unités linguistiques mais de tâches à accomplir dans la langue cible en vue de son acquisition (le 3.1. du Tableau 2); tâches ou opérations, cognitives ou communicatives, ou encore d'apprentissage, peu importe au total, ces contenus centrés 
sur le « comment apprendre? " s'illustrèrent tout particulièrement dans une opération qui fit couler beaucoup d'encre, le « Bangalore project », conduit par N.S. Prabhu (1987).

(iii) Enfin, de l'autre côté de l'échiquier se font jour, surtout de la part des didacticiens français ou francophones, de fortes résistances à l'abandon des contenus linguistiques préalables, qui pourront à l'occasion passer pour conservatrices dès lors qu'elles impliquent une certaine forme de statu quo, même si l'on n'y refuse pas pour autant qu'une attention plus soutenue soit portée aux modalités de l'apprentissage.

Et si peut s'élever (voir Tableau 1) l'invocation rituelle à un supposé cartésianisme français, c'est bien qu'elle fait, au bout du compte, l'affaire des diverses parties. Ici comme repoussoir (décidément, on ne les changera pas...) et là comme armure, ou rempart : impossible, pour un esprit français, d'admettre que l'on puisse enseigner sans savoir ce que l'on va enseigner; pas de salut sans programme; et un programme cela se prépare (à l'avance), cela se programme. Telle est à peu près la position tenue, au sein même des débats d'experts du Conseil de l'Europe à la fin des années quatre-vingt, par des didacticiens aussi autorisés que Denis Girard et Jeanine Courtillon (1988).

Cette position apparaît, chacun l'aura compris, encore plus fortement ancrée s'agissant de FOS. On ne parvient pas, en effet, à y surmonter ce qui a, pour bon nombre de didacticiens français, toutes les apparences d'une contradiction première : quel sens y a-til à parler de besoins et d'objectifs si l'absence de traduction sous forme de contenu prédéterminé interdit tout contrôle de leur plus ou moins grand degré de satisfaction? Pour dire les choses autrement ${ }^{14}$ : comment être sûr de ne pas vendre du Victor Hugo à ceux qui veulent du Marcel Dassault, si les contenus ne sont pas soigneusement définis à l'avance? Une telle attitude pourrait cependant ne plus représenter tout à fait la situation actuelle, tant se multiplient les signes de la distance grandissante prise par les enseignants vis-à-vis des méthodes rigidement organisées et, du coup, à l'égard de contenus du même type ${ }^{15}$.

\subsection{Compromis de raison ou « réponse de Normand " ?}

47 Que penser, finalement, lorsque s'affrontent des positions aussi tranchées? Celle que pour ma part j'ai adoptée depuis quelques années ${ }^{16}$ revêt toutes les apparences d'une tentative de conciliation des contraires. En outre, comme elle n'a pas encore subi l'épreuve des faits par l'expérience de l'enseignement-apprentissage, on ne saurait trop dire ce que vaut un pari n'ayant guère dépassé le stade des postulats de principe.

Quoi qu'il en soit, ancrée dans l'attachement à une certaine forme de programmation susceptible d'assurer le cadrage des objectifs langagiers d'apprentissage, cette position ne peut être tenue pour médiane que dans la mesure où elle conduit à n'adhérer sans restrictions à aucune des propositions alternatives qui inondent aujourd'hui le marché méthodologique.

Rompant, comme on l'a vu, avec la logique hyperlinéaire du système SABOC et de ses divers avatars, elle n'aboutit pas pour autant à disqualifier

50 (i) ni l'identification des besoins, à condition que celle-ci serve à la gestion continue de leurs besoins par les apprenants eux-mêmes, plutôt qu'à la définition d'objectifs et de contenus immuablement gravés dans la cire ;

51 (ii) ni des outils tels que les inventaires et les analyses de situations cibles, pour peu que leur usage soit lui aussi découplé de la stricte détermination de contenus 
immuablement... et vise à alimenter le cours en matériaux ou échantillons langagiers sélectionnés non aléatoirement et de telle sorte qu'ils soient représentatifs des situations et des types de discours réputés requérir une compétence particulière dans la langue cible, en instruments d'évaluation et de régulation du couple besoins/objectifs, eux aussi placés à la libre disposition des apprenants.

Tout cela montre, sans la moindre ambiguïté me semble-t-il, à quel point la question du contenu langagier demeure au centre du débat. Elle nécessiterait beaucoup plus que quelques «réglages » hâtifs; je m'en tiendrai néanmoins, pour finir, à trois points essentiels.

\subsubsection{Des contenus constitués de procédures ou de tâches?}

Les enseignants et didacticiens du FOS prêtent-ils une oreille attentive aux principes et modèles des contenus composés, en matière d'unités, de tâches ou d'activités d'apprentissage ou communicatives - requérant l'usage de la langue cible? On serait tenté de dire: une oreille sans doute mais, les deux, certainement pas; réponse on ne peut plus normande en effet, et qui se décomposera donc en deux.

Tout d'abord, on s'interrogera sur la crédibilité que peut avoir aujourd'hui le syllabus procédural de N.S. Prabhu auprès des didacticiens français, qui n'en ont eu connaissance qu'à travers Prabhu (1987) et l'âpre polémique longuement étalée, dans Applied Linguistics et ELT Journal notamment. Les didacticiens de l'anglais, déplacés naguère à Bangalore en cohortes aussi serrées que choisies, mesureront la frustration et le scepticisme qui saisissent leurs collègues français au vu des informations livrées par l'ouvrage de 1987 : quel progrès, en effet, y a-t-il à définir le contenu en termes de tâche à accomplir plutôt qu'en termes d'unités langagières, lorsque la situation de communication ou la tâche ellemême - décider d'un voyage en train à partir d'un tableau indicateur, par exemple sont si stéréotypées qu'elles ne laissent aucune place à une créativité langagière qui est le fondement même de la compétence de communication? D'autre part, sur le même exemple, et pour rappeler des vérités premières autrefois épinglées par Roulet : chercher l'heure d'arrivée d'un train que l'on ne prendra pas est-il plus formateur, d'un point de vue communicatif, que demander du feu à un laboratoire de langue?

Mais faut-il pour autant jeter le bébé avec l'eau du bain? Tâche et activité occupent dorénavant en FOS une place qui, pour n'être pas nécessairement marquée comme relevant de l'affichage explicite du contenu, ne cesse de croître. D'abord, parce que la pratique de la simulation globale connaît une vogue qui doit sans doute beaucoup à quelques précurseurs, notamment du BELC (les Debyser, Caré, Yaïche, et d'autres encore). Maintenant que, l'expérience venant, les règles en sont clairement établies, les écueils repérés, les animateurs talentueux en nombre suffisant, on voit fleurir les simulations à caractère plus nettement professionnel.

D'une façon beaucoup plus générale, à côté de cette technique particulière mais relevant du même ordre de philosophie pédagogique, s'impose peu à peu la nécessité d'alternatives aux pratiques de formation linguistique classiques, disons dans la tradition " des cours de langue "; infligées à des adultes engagés dans la vie professionnelle, elles génèrent de la régression, à coup sûr (s'asseoir derrière des rangées de tables...), des doses variables de frustration, sans doute (parler quand on n'a rien à dire et, pour le reste...), des savoir-faire communicatifs, peut-être. L'une de ces alternatives désirables consiste à " professionnaliser » le cadre et le temps de la formation; à faire en sorte que 
- hors toute idéalisation - l'ambiance, les activités, le recours aux outils, le rapport au savoir, bien loin de ressembler à ce qu'ils sont dans une classe, prennent plutôt les tonalités du travail de laboratoire (à condition qu'il ne soit pas de langue), du bureau d'étude, de l'agence, de la réunion d'expertise, et que sais-je encore. Les modes d'utilisation de l'ordinateur représentent d'ailleurs un puissant révélateur du très profond clivage qui, en matière d'apprentissage, sépare ceci et cela.

\subsubsection{Contenus et contenus}

Revenons une dernière fois à l'idée selon laquelle le FOS perdrait toute spécificité si un minimum de programmation ne venait garantir que la formation proposée soit en adéquation avec les besoins des apprenants. Seule une vision réductrice, archaïque et, pour tout dire, inculte de l'activité d'acquisition-apprentissage peut conduire à conclure que le «principe d'adéquation » fait obligation d'enseigner les types de discours dont la pratique est requise par ou pour ces apprenants ; ce qui importe, en la matière, est que les apprenants soient placés au contact de ces discours et de leurs situations d'usage. En termes de démarche didactique, il s'agit alors de construire un environnement (ou décor, ou paysage) langagier représentatif de ce qui attend l'apprenant à l'issue de son apprentissage, environnement qu'il sera invité à fréquenter librement (en fait plus ou moins librement selon ses habitudes d'apprentissage et, le cas échéant, celles de ses condisciples, et selon les conceptions méthodologiques de l'enseignant). Cet ensemble, échantillonné et représentatif, constitue bel et bien un contenu; simplement ce n'est, pour utiliser la terminologie consacrée, ni un contenu d'enseignement, ni un contenu d'apprentissage. Je me suis habitué depuis bientôt vingt ans ${ }^{17}$ à le nommer, faute de mieux, un «contenu pour l'apprentissage » : si l'expression n'est pas de celles qui font carrière dans le slogan, elle n'en désigne pas moins exactement l'objet en question.

Une telle pratique situe clairement la question des contenus dans une problématique « de l'abondance ", par opposition à la problématique «du petit pas » ou «de la pénurie » (selon qu'on la loue ou qu'on la stigmatise), qui caractérise généralement les contenus prédéfinis. Cette pédagogie de l'abondance n'est, tout compte fait, que le fruit d'un réalisme hérité, notamment, des travaux acquisitionnistes : on n'y fait pas mine d'ignorer que tout apprenant ne fait jamais son miel que là où il l'entend et comment il l'entend.

Mais comme elle invite aussi à ce que les apprenants introduisent eux-mêmes des éléments de contenu, notamment à l'occasion de leurs tentatives de communication en langue cible, cette conception du « contenu pour l'apprentissage » relève également des approches communicatives de seconde ou de dernière (?) génération ${ }^{18}$, ou encore de toute forme de pédagogie se donnant pour moteur l'interaction communicative entre les participants engagés dans l'activité d'enseignement-apprentissage. Peu importe au total la dénomination : de toute pédagogie où l'acquisition des savoir-faire a sa place au sein du cours, dans le temps et le lieu de l'apprentissage, et pas seulement en dehors, ce dernier étant voué à la seule acquisition de savoirs linguistiques ou métalinguistiques.

\subsubsection{La composante culturelle dans les contenus}

60 Il s'agit là d'un point tout à fait primordial, si ce n'est même le préalable à tous les autres ; mais comme je ne cesse d'y revenir depuis quatre ans, je m'abstiendrai de le développer ici encore. Bien maigre secours, en effet, que des rudiments de grammaire et de vocabulaire, quand la complète méconnaissance des habitudes culturelles de l'Autre vient 
ruiner toute possibilité de compréhension dans les relations de travail ou d'affaires et les échanges scientifiques ou technologiques.

61 Le problème n'est nullement de trouver des exemples d'obstacles culturels à la communication exolingue dans le travail, alors que se multiplient recherches sur les cultures d'entreprise et travaux de sociologie interculturelle ${ }^{19}$. Il est d'abord de bousculer des chapes de représentations chevillées à l'esprit des enseignants (tout au moins des enseignants français de langues) comme (me semble-t-il) à celui de beaucoup d'apprenants, les portant à tenir la science et la culture, les langues comme véhicule de l'une et comme véhicule de l'autre, pour territoires hermétiquement étanches, aliénés, antagonistes, inconciliables, irréconciliables. C'est au prix de cette forme (quelque peu ironique) d'acculturation que la composante culturelle de la relation à l'étranger, dont la vague se fait aujourd'hui lame de fond s'agissant d'apprentissage de la « langue usuelle », recevra droit de cité dans les contenus des cours de FOS.

Quant aux outils et dispositifs susceptibles de permettre cette intégration, ils ne font pas non plus défaut. On se limitera, pour finir, à la seule mention de deux réalisations déjà bien connues et du plus grand intérêt pour la manière dont elles articulent, chacune à sa manière, langue et culture, en une configuration polymorphe où des syllabus divers composent le curriculum d'enseignement-apprentissage: d'une part les travaux de Michael Byram et, d'autre part, le « curriculum multidimensionnel canadien $»^{20}$.

\section{BIBLIOGRAPHIE}

AUPELF-Crédif, éds. 1982. Transferts de formation. Paris : AUPELF.

Béacco, J.-Cl. et D. Lehmann (dir.). 1990. Publics spécifiques et communication spécialisée. Paris : Hachette (Coll. F-Recherches et applications).

Brumfit, C. \& K. Johnson (dir.). 1979. The Communicative Approach to Language Teaching. Oxford : Oxford University Press.

Byram, M. 1992. Culture et éducation en langue étrangère. Paris : Hatier/Didier-Crédif (Coll. LAL).

Couillerot, J. et H. Farid. 1990. « Français sur objectifs spécifiques. Parier sur l'ouverture ». Le français dans le monde 235.

Girard, D., J. Courtillon, B. Page et R. Richterich. 1988. Choix et distribution des contenus dans les programmes de langues. Strasbourg: Conseil de l'Europe.

Hutchinson, T. \& A. Waters. 1987. English for Specific Purposes. A learning-centred approach. Cambridge : Cambridge University Press (Coll. New Directions in Language Teaching).

LeBlanc, R. 1989. « Le curriculum multidimensionnel : une approche intégrée pour l'enseignement de la langue seconde ». Études de linguistique appliquée 75.

LeBlanc, R. 1995. «Le curriculum multidimensionnel : questions d'implémentation ». Études de linguistique appliquée 98. 
Lehmann, D. 1993. Objectifs spécifiques en langue étrangère. Les programmes en question. Paris : Hachette (Coll. F-Références).

Mariet, F. 1980. « Les armes égales que sont les mots. Sur la dimension sociologique de la rhétorique des sciences économiques ». In Lehmann D. (dir.), Lecture fonctionnelle de textes de spécialité. Paris : Didier (Coll. VIC).

Prabhu, N.S. 1987. Second Language Pedagogy. Oxford : Oxford University Press (Coll. Applied Linguistics),

Trim, J. (dir.). 1973. Systèmes d'apprentissage des langues vivantes par les adultes. Strasbourg : Conseil de l'Europe.

Trimble, L. 1985. English for Science and Technology. A discourse approach. Cambridge : Cambridge University Press.

\section{NOTES}

1. Le présent article est le texte remanié de la conférence plénière prononcée par l'auteur lors du $18^{\mathrm{e}}$ colloque du GERAS à l'ENS Ulm de Paris en mars 1997, dont le thème était Langue anglaise de spécialité/langue française de spécialité.

2. Volontairement non référencés, ils émanent surtout de «décideurs" français, parfois de didacticiens français (2 et 4), parfois de partenaires étrangers (5).

3. Pour un exposé des conditions et effets de cette prégnance, se reporter au chapitre 3 de Lehmann (1993).

4. Voir les interventions de B. Aubert à la table ronde concluant Béacco et Lehmann, dir. (1990).

5. AUPELF-Crédif, éds (1982).

6. C'est ainsi qu'un excellent matériel expérimental réalisé par deux collègues de Paris 8 et Paris 10 est resté dans les cartons faute de trouver éditeur à... sa taille.

7. Puisque l'on parle à son propos d'une «École de Lancaster ", illustrée notamment, outre les précédents, par les plumes de Breen, Candlin ou encore Allwright.

8. J'ai, pour ma part (1993), concentré sur le seul Trimble un tir présentant l'avantage décisif d'être bien trop court pour atteindre jamais une telle cible, tout en me préservant des foudres de mes collègues Linkstiks ou Philogs français.

9. Aussi ai-je cédé à la commodité de désigner ce modèle par la formule "système $S A B O C$ ».

10. E. Roulet et l'équipe du Crédif animée par D. Coste : J. Courtillon, M. Martins-Baltar, E. Papo.

11. Il n'est certainement pas indifférent de noter que ces deux groupes avaient en commun d'être principalement constitués d'anglicistes.

12. Voir, à côté d'une prise de distance qui ne se démentira pas chez Widdowson, les nombreuses publications, collectives ou non, de la fin des années 1970. Notamment, Brumfit \& Johnson, dir. (1979).

13. On est censé y voir une allusion à ces immortels compagnons des veillées anglo-saxonnes que furent les disques de la série « Sing along with Mitch».

14. Par référence au bouquet d'opinions (en l'occurrence la citation 4) sur la diffusion du français présenté au début de cet exposé.

15. À l'appui de ce qui n'est pas qu'un sentiment: à l'occasion d'un DEA entrepris sous ma direction, Caroline Chevalier montre, recensement à l'appui, que bon nombre des manuels récents de français des affaires n'affichent explicitement aucun contenu syntaxique spécifique.

16. Elle apparaissait déjà pour l'essentiel dans l'avant-dernier chapitre de Lehmann (1993), notamment 7.3., 192 et sq. 
17. Depuis que, dans le cadre d'un projet mené au sein du Crédif, le sociologue François Mariet en construisit un premier et magistral exemplaire, selon des principes largement empruntés à P. Bourdieu ; voir Mariet (1980).

18. Du moins Christopher Brumfit (dans Brumfit \& Johnson, dir. [1979], 183) considère-t-il l'apport de contenu par les apprenants comme «l'impact le plus durable du mouvement communicatif » (c'est moi qui traduis).

19. Pour une première information, Lehmann (1993), chapitre 1.

20. Pour le second, on se reportera à Leblanc et, si l'on souhaite plus de détails, aux six ouvrages non référencés publiés en 1990, sous la direction du même LeBlanc, par l'Association canadienne des professeurs de langues secondes et M Éditeur.

\section{RÉSUMÉS}

L'état comparatif de l'ESP et du FOS amène d'abord à pointer la totale disproportion existant entre les deux marchés linguistiques et éditoriaux; puis à souligner le poids tout particulier des facteurs «politiques" en FOS. Autre asymétrie, encore, que l'influence persistante des thèses anglo-saxonnes sur les positions françaises au plan méthodologique. Schématiquement décrit, celui-ci se caractérise dans les deux secteurs par la coexistence de trois mêmes courants, toujours concurrents bien qu'inégalement porteurs. Mais si l'on s'accorde assez bien aujourd'hui pour se défier de démarches trop systématiques et prêter une attention croissante aux modalités de l'apprentissage, les didacticiens français demeurent, quant à eux, particulièrement sensibles à la nécessité méthodologique d'un contenu d'enseignement préalable - défini en termes linguistiques et/ou non linguistiques - en vue de ménager la diversité des besoins et des objectifs.

A comparison of ESP and its equivalent for the French language (FSP) highlights the disproportion between the two markets, in both linguistic and editorial terms, also the fact that "political" factors play a part in FSP. A further difference is the continued influence of AngloSaxon arguments on French methodology. This is described briefly, noting that in both sectors are found the same three trends, though in varying degrees of intensity. There is now agreement over the fact that methods which are too systematic are to be avoided, and more attention should be paid to modalities of language learning. Nevertheless, French didacticians remain particularly sensitive to the methodological necessity for having a pre-determined programme content defined in linguistic and/or non-linguistic term - in order to deal with the diverse needs and objectives of learners.

\section{INDEX}

Mots-clés : besoins, centration sur la langue, centration sur l'apprenant, composante culturelle, curriculum, ESP, FOS, situation-cible, syllabus, système

Keywords : cultural component, curriculum, ESP, FSP, language-centred approach, learnercentred approach, need, syllabus, system, target-situation 
AUTEUR

DENIS LEHMANN 